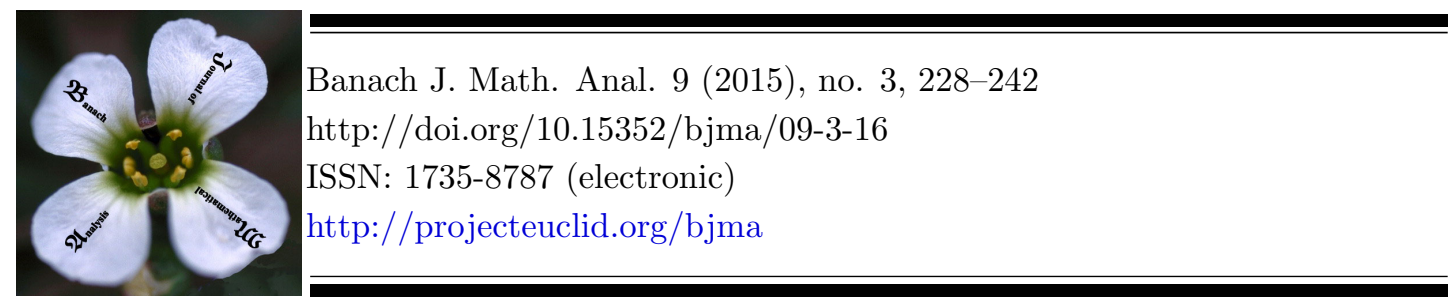

\title{
GENERALIZATION OF SHARP AND CORE PARTIAL ORDER USING ANNIHILATORS
}

\author{
DRAGAN S. RAKIĆ \\ Communicated by M. Brešar
}

\begin{abstract}
The sharp order is a well known partial order defined on the set of complex matrices with index less or equal one. Following Šemrl's approach, Efimov extended this order to the set of those bounded Banach space operators $A$ for which the closure of the image and kernel are topologically complementary subspaces. In order to extend the sharp order to arbitrary ring $R$ (particulary to Rickart and Rickart *-rings) we use the notions of annihilators. The concept of the sharp order is extended to the set $\mathcal{I}_{R}$ of those elements for which left and right annihilators are respectively principal left and principal right ideals generated by the same idempotent. It is proved that the sharp order is a partial order relation on $\mathcal{I}_{R}$. Following the idea we also extend and discuss the recently introduced concept of core partial order.
\end{abstract}

\section{INTRODUCTION AND PRELIMINARIES}

Let $M_{n}$ be the algebra of all $n \times n$ complex matrices and let

$$
I_{1, n}=\left\{A \in M_{n}: \operatorname{rank} A=\operatorname{rank} A^{2}\right\} .
$$

For any $A \in M_{n}$ there exists $A^{(1)} \in M_{n}$ such that $A A^{(1)} A=A$ and in this case $A^{(1)}$ is called the inner inverse of $A$. But, there exists the matrix $A^{\sharp} \in M_{n}$ such that

$$
A A^{\sharp} A=A, \quad A^{\sharp} A A^{\sharp}=A, \quad A A^{\sharp}=A^{\sharp} A
$$

Date: Received: Aug. 14, 2014; Accepted: Nov. 8, 2014.

2010 Mathematics Subject Classification. Primary 47C10; Secondary 06A06, 15A09, 16 U99.

Key words and phrases. Sharp partial order, core partial order, linear bounded operator, Rickart ring, annihilator. 
if and only if $A \in I_{1, n}$. When $A^{\sharp}$ exists then it is unique and it is called the group inverse of $A$, [2]. The minus partial order on $M_{n}$ introduced by Hartwig in [8] is defined by:

$$
A \leq^{-} B \Longleftrightarrow A A^{(1)}=B A^{(1)}, A^{(1)} A=A^{(1)} B \text { for some inner inverse } A^{(1)} .
$$

Another equivalent definition is:

$$
A \leq^{-} B \Longleftrightarrow \operatorname{rank} B=\operatorname{rank} A+\operatorname{rank}(B-A) .
$$

The well known sharp partial order on $I_{1, n}$, introduced by Mitra in [14], is defined by:

$$
A \leq^{\sharp} B \Longleftrightarrow A A^{\sharp}=B A^{\sharp} \text { and } A^{\sharp} A=A^{\sharp} B .
$$

If $X$ is normed space then the set of all bounded linear operators on $X$ is denoted by $\mathcal{B}(X)$. The image and kernel of $A \in \mathcal{B}(X)$ are denoted by $\operatorname{Im} A$ and Ker $A$ respectively. Of course, if $Y \subseteq X$ then $\bar{Y}$ is the norm closure of $Y$. By the direct sum we mean the topological direct sum. Let $M$ and $N$ be subspaces of normed space $X$. Recall that $X=M \oplus N$ is topological direct sum if and only if there exists a bounded idempotent $P \in \mathcal{B}(X)$ such that $\operatorname{Im} \mathrm{P}=\mathrm{M}$ and Ker $\mathrm{P}=\mathrm{N}$. In this case $M$ and $N$ are closed in $X$. The subspace $M$ is said to be complemented in $X$ if there exists subspace $N$ such that $X=M \oplus N$, that is if $M=\operatorname{Im} \mathrm{P}$ for some bounded idempotent $P \in \mathcal{B}(X)$. In that case we say that $M$ and $N$ are complementary subspaces. Suppose that $X_{1}, \ldots, X_{n}$ are subspaces of Banach space $X$. Recall that $X=X_{1} \oplus \cdots \oplus X_{n}$ is topological direct sum if and only if it is algebraic direct sum and $X_{i}$ is closed in $X$ for all $i=1,2, \ldots, n$. Identifying matrices with operators acting on $\mathbb{C}^{n}$ we can write $M_{n}=\mathcal{B}\left(\mathbf{C}^{n}\right)$. If $X$ is Banach space then $A \in \mathcal{B}(X)$ has an inner inverse if and only if $\operatorname{Im} A$ is closed and complemented in $X$ and $\operatorname{Ker} A$ is complemented in $X$ (see [5]).

In [16] Šemrl extended the notion of minus partial order from $M_{n}$ to $\mathcal{B}(H)$, where $H$ is a Hilbert space. Since he did not want to restrict the definition to the closed range operators and since the notion of rank can not be applied when $H$ is infinite dimensional, he introduced the following definition.

Definition 1.1. [16] Let $H$ be a Hilbert space. For $A, B \in \mathcal{B}(H)$ we write $A \leq^{-} B$ if and only if there exist idempotent operators $P, Q \in \mathcal{B}(H)$ such that

$$
\operatorname{Im} P=\overline{\operatorname{Im} A}, \quad \operatorname{Ker} Q=\operatorname{Ker} A, \quad P A=P B, \quad A Q=B Q .
$$

Šemrl proved that his relation coincides with Hartwig's minus partial order when $\operatorname{dim} X<\infty$ and that $\leq^{-}$is a partial order relation on $\mathcal{B}(H)$.

Following Šemrl's approach, Efimov [7] extended the notion of sharp order to $\mathcal{B}(X)$ the algebra of bounded linear operators on Banach space $X$. Let

$$
I_{X}=\{A \in \mathcal{B}(X): \overline{\operatorname{Im} A} \oplus \operatorname{Ker} A=X\} .
$$

It is easy to see that $A \in I_{X}$ if and only if there exists idempotent $P_{A} \in \mathcal{B}(X)$ such that

$$
\operatorname{Im} P_{A}=\overline{\operatorname{Im} A} \text { and } \operatorname{Ker} P_{A}=\operatorname{Ker} A .
$$

Furthermore, such idempotent $P_{A} \in \mathcal{B}(X)$ is unique and

$$
A=P_{A} A=A P_{A} \text {. }
$$


As we can see $I_{X}$ coincides with $I_{1, n}$ when $\operatorname{dim} X<\infty$. The Efimov's definition of the sharp order on $\mathcal{B}(X)$ is as follows.

Definition 1.2. [7] Let $X$ be a Banach space. For $A, B \in \mathcal{B}(X)$ we say that $A \leq \sharp B$ if $A \in I_{X}$ and

$$
A=P_{A} B=B P_{A}
$$

The sharp order $\leq^{\sharp}$ is a partial order relation on $I_{X},[7]$ and this relation is an extension of the sharp partial order from $I_{1, n}$ to $I_{X}$.

Our aim is to extend the sharp order to more general rings. Recently, the sharp order has been generalized to the subset of all elements in a general ring with identity which have the group inverse, see [12]. We do not want to restrict our attention to elements which possess the group inverse, and of course we can not use the notions such as image or kernel. Instead, we will use the notion of annihilators.

Let $R$ be a ring with the unit 1 . An element $p \in R$ is an idempotent if $p^{2}=p$. If $R$ has an involution then idempotent $p \in R$ is self-adjoint if $p^{*}=p$. For $S \subseteq R$ the right annihilator $S^{\circ}$ of $S$ is defined by

$$
S^{\circ}=\{x \in R: s x=0 \text { for all } s \in S\} .
$$

Similarly,

$$
{ }^{\circ} S=\{x \in R: x s=0 \text { for all } s \in S\}
$$

denotes the left annihilator of $S . S^{\circ}$ is a right ideal of $R$ and ${ }^{\circ} S$ is a left ideal of $R$. If $S=\{a\}$ then we shortly write $a^{\circ}$ and ${ }^{\circ} a$ instead of $\{a\}^{\circ}$ and ${ }^{\circ}\{a\}$. A ring $R$ is Rickart ring if for every $a \in R$ there exist idempotents $p, q \in R$ such that $a^{\circ}=p R$ and ${ }^{\circ} a=R q$. Every Rickart ring has the identity element 1 [10]. If $R$ is a ring with an involution then $R$ is Rickart $*$-ring if for every $a \in R$ there exist self-adjoint idempotents $p, q \in R$ such that $a^{\circ}=p R$ and ${ }^{\circ} a=R q$. Note that $p$ and $q$ are unique.

The main result in Section 2 is Theorem 2.4 where the following result is proved. If $A \in \mathcal{B}(X)$ where $X$ is a normed vector space then the left annihilator of $A$ is the principal left ideal generated by an idempotent if and only if $\overline{\operatorname{Im} A}$ is complemented in $X$. Also, the right annihilator of $A$ is the principal right ideal generated by an idempotent if and only if Ker A is complemented in $X$. This result allows us to characterize the set $I_{X}$ without the use of the notions of image and kernel and thus we will obtain the equivalent algebraic definition of the sharp order on $\mathcal{B}(X)$. Using the results from Section 2, in Section 3 we extend the notion of the sharp order to an arbitrary ring $R$. For an arbitrary ring $R$ we define the set

$$
\mathcal{I}_{R}=\left\{a \in R: a^{\circ}=q R,{ }^{\circ} a=R q \text { for some idempotent } q \in R\right\},
$$

which coincides with $I_{X}$ when $R=\mathcal{B}(X)$. If such an idempotent $q$ exists, then it is unique. Now, for $a, b \in \mathcal{I}_{R}$ we write:

$$
a \leq^{\sharp} b \Longleftrightarrow a=b p=p b,
$$

where $p=1-q$. We will show that $\leq \sharp$ is a partial order relation on $\mathcal{I}_{R}$. We will also generalize the notions of left sharp and right sharp orders and we will 
show that these relations are partial orders on $R$. In the last section we extend the recently introduced notion of the core partial order [1] combining the sharp and the star partial order [6]. A special case when $R=\mathcal{B}(X)$ will be discussed separately.

\section{EquiVALENT DEFINITION OF THE SHARP PARTIAL ORDER}

In this section we will give a new equivalent algebraic definition of the sharp partial order on $\mathcal{B}(X)$ where $X$ is a Banach space. Let

$$
\mathcal{I}_{X}=\left\{A \in \mathcal{B}(X):{ }^{\circ} A={ }^{\circ} P \text { and } A^{\circ}=P^{\circ} \text { for some idempotent } P \in \mathcal{B}(X)\right\} \text {. }
$$

To prove that the idempotent $P$ appearing in (2.1) is unique, we give the following lemma but in a broader context of unitary ring $R$.

Lemma 2.1. Let $p$ and $q$ be idempotents in $R$. If $p^{\circ} \subseteq q^{\circ}$ and ${ }^{\circ} q \subseteq{ }^{\circ} p$ then $p=q$.

Proof. From $p^{\circ} \subseteq q^{\circ}$ we obtain $q(1-p)=0$ that is $q=q p$. From ${ }^{\circ} q \subseteq{ }^{\circ} p$ we obtain $(1-q) p=0$ so $p=q p=q$.

When $A \in \mathcal{I}_{X}$, we will denote the unique idempotent $P$ appearing in (2.1) by $P_{A}$.

Definition 2.2. Let $X$ be a Banach space. For $A, B \in \mathcal{B}(X)$ we say that $A \leq \sharp B$ if $A \in \mathcal{I}_{X}$ and $A=P_{A} B=B P_{A}$.

To prove that the relations given by Definition 1.2 and Definition 2.2 are equal we need the following lemma and theorem.

Lemma 2.3. If $p \in R$ is idempotent then $R(1-p)={ }^{\circ} p$ and $(1-p) R=p^{\circ}$.

Proof. The proof is straightforward, see Theorem 2 in [10].

Theorem 2.4. Let $X$ be the normed space. Let $P \in \mathcal{B}(X)$ be an idempotent operator and $A \in \mathcal{B}(X)$. Then:

(1) $A^{\circ}=(I-P) \mathcal{B}(X) \Longleftrightarrow A^{\circ}=P^{\circ} \Longleftrightarrow \operatorname{Ker} P=\operatorname{Ker} A$;

$(2)^{\circ} A=\mathcal{B}(X)(I-P) \Longleftrightarrow{ }^{\circ} A={ }^{\circ} P \Longleftrightarrow \operatorname{Im} P=\overline{\operatorname{Im} A}$.

Proof. By Lemma 2.3, we know that $A^{\circ}=(I-P) \mathcal{B}(X)$ if and only if $A^{\circ}=P^{\circ}$ and ${ }^{\circ} A=\mathcal{B}(X)(I-P)$ if and only if ${ }^{\circ} A={ }^{\circ} P$.

(1): Suppose that $A^{\circ}=(I-P) \mathcal{B}(X)$ that is $A^{\circ}=P^{\circ}$. We have $A(I-P)=0$ so Ker $P=\operatorname{Im}(I-P) \subseteq \operatorname{Ker} A$. On the other hand suppose that $A x_{0}=0$ for some $x_{0} \in X, x_{0} \neq 0$. By the consequence of the Hahn-Banach theorem there exists a bounded linear functional $f: X \rightarrow \mathbb{C}$ with $f\left(x_{0}\right)=1$. Let $S: X \rightarrow X$ be the map defined by $S x=f(x) x_{0}, x \in X$. Then $S \in \mathcal{B}(X)$ with $\|S\|=\|f\|\left\|x_{0}\right\|$. For any $x \in X$ we have $A S x=A\left(f(x) x_{0}\right)=f(x) A x_{0}=0$ so $S \in A^{\circ}$. Therefore $S \in P^{\circ}$ and hence $0=P S x_{0}=P\left(f\left(x_{0}\right) x_{0}\right)=P x_{0}$, so $x_{0} \in \operatorname{Ker} P$. Thus $\operatorname{Ker} A=\operatorname{Ker} P$.

Now suppose that $\operatorname{Ker} P=\operatorname{Ker} A$. Note that $A^{\circ}=P^{\circ}$ if and only if for all $C \in \mathcal{B}(X)$ the following equivalence holds:

for every $x \in X(A C x=0) \Longleftrightarrow$ for every $x \in X(P C x=0)$. 
Since for all $z \in X, A z=0$ if and only if $P z=0$, it follows that $A^{\circ}=P^{\circ}=$ $(I-P) \mathcal{B}(X)$.

(2): Suppose that ${ }^{\circ} A=\mathcal{B}(X)(I-P)$ that is ${ }^{\circ} A={ }^{\circ} P$ and let us prove that $\operatorname{Im} P=\overline{\operatorname{Im} A}$. We have $(I-P) A=0$ so

$$
\overline{\operatorname{Im} A} \subseteq \operatorname{Ker}(I-P)=\operatorname{Im} P,
$$

since $\operatorname{Im} P$ is closed. It follows that with respect to direct sum decomposition $X=\operatorname{Im} P \oplus \operatorname{Ker} P, A, P: \operatorname{Im} P \oplus \operatorname{Ker} P \rightarrow \operatorname{Im} P \oplus \operatorname{Ker} P$ have the following matrix representations

$$
A=\left[\begin{array}{cc}
A_{1} & A_{2} \\
0 & 0
\end{array}\right] \text { and } P=\left[\begin{array}{ll}
I & 0 \\
0 & 0
\end{array}\right],
$$

where $A_{1}: \operatorname{Im} P \rightarrow \operatorname{Im} P$ and $A_{2}: \operatorname{Ker} P \rightarrow \operatorname{Im} P$ are bounded linear operators. Suppose, contrary to our claim, that there exists

$$
x_{0} \in \operatorname{Im} P \backslash \overline{\operatorname{Im} A} .
$$

Of course, $x_{0} \neq 0$. By the consequence of the Hahn-Banach theorem there exists a bounded linear functional $f: \operatorname{Im} P \rightarrow \mathbb{C}$ with $f\left(x_{0}\right)=1$ and $f(x)=0$ for all $x \in \operatorname{Im} A$. Let $S_{1}: \operatorname{Im} P \rightarrow \operatorname{Im} P$ be the map defined by $S_{1}(x)=f(x) x_{0}$. It is easy to show that $S_{1}$ is bounded linear operator with $S_{1} x=0$ for all $x \in \operatorname{Im} A$ and $S_{1} x_{0}=x_{0} \neq 0$. Consequently, since $\operatorname{Im} A_{1} \subseteq \operatorname{Im} A$ and $\operatorname{Im} A_{2} \subseteq \operatorname{Im} A$ we obtain $S_{1} A_{1}=0$ and $S_{1} A_{2}=0$. Finally, let

$$
S=\left[\begin{array}{cc}
S_{1} & 0 \\
0 & 0
\end{array}\right]
$$

Of course, $S$ is linear and bounded and $S x_{0}=S_{1} x_{0}=x_{0} \neq 0$. We have

$$
S A=\left[\begin{array}{cc}
S_{1} A_{1} & S_{1} A_{2} \\
0 & 0
\end{array}\right]=0 \text { and } S P=\left[\begin{array}{cc}
S_{1} & 0 \\
0 & 0
\end{array}\right]=S \neq 0 .
$$

This contradicts our assumption. We have proved that $\operatorname{Im} P=\overline{\operatorname{Im} A}$.

Suppose now that $\operatorname{Im} P=\overline{\operatorname{Im} A}$. Thus $\operatorname{Im} A \subseteq \operatorname{Ker}(I-P)$ so $(I-P) A=0$ that is $A=P A$. This gives ${ }^{\circ} P \subseteq{ }^{\circ} A$. On the other hand, suppose that $C A=0$ for some $C \in \mathcal{B}(X)$ and let $x \in X$ be arbitrary. As $P x \in \operatorname{Im} P=\overline{\operatorname{Im} A}$, there exists a sequence $\left(x_{n}\right)_{n \in \mathbb{N}} \subseteq X$ such that $A x_{n}$ converges in norm to $P x$. Since $C$ is continuous operator it follows that $C A x_{n} \rightarrow C P x$. But $C A x_{n}=0$ for all $n \in \mathbb{N}$ so $C P x=0$. This is valid for all $x \in X$ so $C P=0$. We have proved that ${ }^{\circ} A={ }^{\circ} P$.

Remark 2.5. Recall that if $M$ is closed subspace of a Hilbert space $X$ then $M$ is complemented in $X$; namely $X=M \oplus M^{\perp}$. In other words there exists self-adjoint bounded idempotent operator $P \in \mathcal{B}(X)$ with $\operatorname{Im} P=M$. As we know if $X$ is Banach space then closed subspaces of $X$ is not complemented in general. Furthermore, $\operatorname{Ker} A$ is closed for every bounded operator. According to this remark, as a direct consequence of Theorem 2.4 we obtain the following important well known result. If $X$ is a Hilbert space then $\mathcal{B}(X)$ is Rickart $*$-ring. But, if $X$ is a Banach space then $\mathcal{B}(X)$ is not Rickart ring in general.

Theorem 2.6. Let $X$ be a Banach space. Then: 
(1) $I_{X}=\mathcal{I}_{X}$

(2) The sharp partial order given by Definition 1.2 and the relation given by Definition 2.2 are equal.

Proof. The proof is a direct consequence of Lemma 2.3 and Theorem 2.4.

\section{Sharp PARTIAL ORDER IN RING}

Let $R$ be an arbitrary ring with identity 1 . Definition of the set $\mathcal{I}_{X}$ and sharp order given by Definition 2.2 make sense in $R$. Let

$$
\mathcal{I}_{R}=\left\{a \in R:{ }^{\circ} a={ }^{\circ} p \text { and } a^{\circ}=p^{\circ} \text { for some idempotent } p \in R\right\} .
$$

We proved the uniqueness of $p$ in Lemma 2.1 so we can and we will write $p=p_{a}$. If $a \in \mathcal{I}_{R}$ then $\left(1-p_{a}\right) a=a\left(1-p_{a}\right)=0$ since $1-p_{a} \in{ }^{\circ} p_{a} \cap p_{a}^{\circ}$. Hence

$$
a=p_{a} a=a p_{a} .
$$

Definition 3.1. For $a, b \in R$ we say that $a \leq^{\sharp} b$ if $a \in \mathcal{I}_{R}$ and

$$
a=p_{a} b=b p_{a} .
$$

In what follows, we need the following remark.

Remark 3.2. Suppose that $1=e_{1}+e_{2}+e_{3}$ and $1=r_{1}+f_{2}+f_{3}$ are two decompositions of the identity of $R$ (we say that $1=e_{1}+e_{2}+e_{3}$ is decomposition of the identity of the ring $R$ if $e_{1}, e_{2}$ and $e_{3}$ are idempotents with $e_{i} e_{j}=0$, for $i \neq j$ ). Then for any $x \in R$ we have

$$
x=1 \cdot x \cdot 1=\left(e_{1}+e_{2}+e_{3}\right) x\left(f_{1}+f_{2}+f_{3}\right)=\sum_{i, j=1}^{3} e_{i} x f_{j},
$$

which can be rewritten in the matrix form

$$
x=\left[\begin{array}{lll}
x_{11} & x_{12} & x_{13} \\
x_{21} & x_{22} & x_{23} \\
x_{31} & x_{32} & x_{33}
\end{array}\right]_{e \times f}
$$

where $x_{i j}=e_{i} x f_{j}$. One can see that (3.1) represents $R$ as a direct sum of abelian groups $e_{i} R f_{j}:=\left\{e_{i} x f_{j}: x \in R\right\}$ :

$$
R=\bigoplus_{i, j=1}^{3} e_{i} R f_{j} .
$$

Let $y=\left[y_{i j}\right]_{e \times f}$ and $z=\left[z_{i j}\right]_{f \times e}$. As $e_{i} e_{j}=0$ and $f_{i} f_{j}=0$ for $i \neq j$, we can interpreted the usual algebraic operations $x+y$ and $x z$ in $R$ as simple operations between appropriate $3 \times 3$ matrices over $R$. The decomposition of the identity $1=e_{1}+e_{2}+e_{3}$ is orthogonal if $e_{i}, i=1,2,3$ are self-adjoint idempotents. Furthermore, it is easy to see that

$$
x^{*}=\left[\begin{array}{lll}
x_{11}^{*} & x_{21}^{*} & x_{31}^{*} \\
x_{12}^{*} & x_{22}^{*} & x_{32}^{*} \\
x_{13}^{*} & x_{23}^{*} & x_{33}^{*}
\end{array}\right]_{f^{*} \times e^{*}},
$$


with respect to decompositions $1=f_{1}^{*}+f_{2}^{*}+f_{3}^{*}$ and $1=e_{1}^{*}+e_{2}^{*}+e_{3}^{*}$. When $e_{i}=f_{i}$, the decomposition (3.1) is known as the two-sided Peirce decomposition of the ring $R,[9]$.

When we have two idempotents $p, q \in R$ then for $x \in R$ we can write

$$
x=\left[\begin{array}{ll}
x_{11} & x_{12} \\
x_{21} & x_{22}
\end{array}\right]_{p \times q},
$$

where $x_{11}=p x q, x_{12}=p x(1-q), x_{21}=(1-p) x q, x_{22}=(1-p) x(1-q)$.

Lemma 3.3. Let $a, b \in \mathcal{I}_{R}$. Then the following statements are equivalent:

(1) $a=p_{a} b$;

(2) $a^{2}=a b$.

Any of (1) or (2) implies $p_{a} p_{b}=p_{a}$.

Proof. Since $a=p_{a} a$ we have

$$
\begin{aligned}
a=p_{a} b & \Longleftrightarrow p_{a}(b-a)=0 \Longleftrightarrow b-a \in p_{a}^{\circ} \Longleftrightarrow b-a \in a^{\circ} \\
& \Longleftrightarrow a(b-a)=0 \Longleftrightarrow a^{2}=a b .
\end{aligned}
$$

Suppose that $a=p_{a} b$. From $1-p_{b} \in p_{b}^{\circ}=b^{\circ}$ we obtain $a\left(1-p_{b}\right)=p_{a} b\left(1-p_{b}\right)=0$ so $1-p_{b} \in a^{\circ}=p_{a}^{\circ}$. Thus $p_{a}\left(1-p_{b}\right)=0$.

By duality, we can obtain the following lemma.

Lemma 3.4. Let $a, b \in \mathcal{I}_{R}$. Then the following statements are equivalent:

(1) $a=b p_{a}$;

(2) $a^{2}=b a$.

Any of (1) or (2) implies $p_{b} p_{a}=p_{a}$.

Theorem 3.5. Let $a, b \in \mathcal{I}_{R}$. Then the following statements are equivalent:

(1) $a \leq \sharp b$;

(2) There exists a decomposition of the identity of the ring $R, 1=f_{1}+f_{2}+f_{3}$, where $f_{1}=p_{a}$ and $f_{2}=p_{b-a}$ such that

$$
a=\left[\begin{array}{lll}
a & 0 & 0 \\
0 & 0 & 0 \\
0 & 0 & 0
\end{array}\right]_{f \times f} \text { and } b=\left[\begin{array}{ccc}
a & 0 & 0 \\
0 & b-a & 0 \\
0 & 0 & 0
\end{array}\right]_{f \times f} \text {. }
$$

(3) $a^{2}=a b=b a$.

Proof. (1) $\Rightarrow(2)$ : Suppose that $a, b \in \mathcal{I}_{R}$ and $a \leq^{\sharp} b$. Then $a=p_{a} b=b p_{a}$. By lemmas 3.3 and 3.4 we have

$$
p_{a}=p_{a} p_{b}=p_{b} p_{a}
$$

It follows that $p_{b}-p_{a}$ is an idempotent. We proceed to show that $b-a \in \mathcal{I}_{R}$ and

$$
p_{b-a}=p_{b}-p_{a}
$$

Suppose that $\left(p_{b}-p_{a}\right) x=0$ for some $x \in R$. Then

$$
(b-a) x=\left(b p_{b}-b p_{a}\right) x=b\left(p_{b}-p_{a}\right) x=0 .
$$


On the other hand if $(b-a) x=0$ then $b\left(1-p_{a}\right) x=(b-a) x=0$, so $\left(1-p_{a}\right) x \in$ $b^{\circ}=p_{b}^{\circ}$. Thus

$$
0=p_{b}\left(1-p_{a}\right) x=\left(p_{b}-p_{a}\right) x .
$$

Therefore, $(b-a)^{\circ}=\left(p_{b}-p_{a}\right)^{\circ}$. Likewise, ${ }^{\circ}(b-a)={ }^{\circ}\left(p_{b}-p_{a}\right)$. It follows that $b-a \in \mathcal{I}_{R}$ and $p_{b-a}=p_{b}-p_{a}$. Let $f_{1}=p_{a}, f_{2}=p_{b}-p_{a}=p_{b-a}$ and $f_{3}=1-p_{b}$. From (3.3) we obtain that $1=f_{1}+f_{2}+f_{3}$ is a decomposition of the identity of $R$. We know that $a=p_{a} a p_{a}=f_{1} a f_{1}$. The element $a$ (or any other element of the ring) has a unique matrix representation with respect to decomposition of the identity, $1=f_{1}+f_{2}+f_{3}$. Since $a=f_{1} a f_{1}$ it follows that upper left entry of the matrix form of $a$ is equal $a$ and all other entries are equal zero. The representation for $a$ in (3.2) follows. Similarly, the matrix form for $b$ follows from $b-a=p_{b-a}(b-a) p_{b-a}=f_{2}(b-a) f_{2}$, that is $b=a+(b-a)=f_{1} a f_{1}+f_{2}(b-a) f_{2}$.

$(2) \Rightarrow(3)$ is obvious; ordinary matrix multiplication.

$(3) \Rightarrow(1)$ follows by lemmas 3.3 and 3.4 .

By Theorem 3.5 we can characterize the set of all elements $b$ that are above an element $a \in \mathcal{I}_{R}$ under the sharp partial order:

$$
a \leq^{\sharp} b \Longleftrightarrow b=\left[\begin{array}{cc}
a & 0 \\
0 & b_{1}
\end{array}\right]_{p_{a} \times p_{a}} \Longleftrightarrow b=a+\left(1-p_{a}\right) x\left(1-p_{a}\right),
$$

where $b_{1} \in\left(1-p_{a}\right) R\left(1-p_{a}\right)$ and $x \in R$ are arbitrary.

Remark 3.6. Let us see to which representations the matrix forms given in (3.2) reduces in the case $R=\mathcal{B}(X)$ where $X$ is Banach space. Suppose that $A, B \in I_{X}$ and $A \leq \sharp B$. We have proved in Theorem 3.5 that $I=F_{1}+F_{2}+F_{3}$ where $F_{1}=P_{A}, F_{2}=P_{B-A}=P_{B}-P_{A}, F_{3}=I-P_{B}$ is a decomposition of the identity $I$ of $\mathcal{B}(X)$. Note that every decomposition of the identity of $\mathcal{B}(X)$ implies one topological direct sum. Namely, $I=F_{1}+F_{2}+F_{3}$ implies

$$
X=\operatorname{Im} I=\operatorname{Im} F_{1} \oplus \operatorname{Im} F_{2} \oplus \operatorname{Im} F_{3} .
$$

Indeed, since for any $x \in X, x=I x=F_{1} x+F_{2} x+F_{3} x$ we have $X=\operatorname{Im} I=$ $\operatorname{Im} F_{1}+\operatorname{Im} F_{2}+\operatorname{Im} F_{3}$. Furthermore, since $F_{i} F_{j}=0$ we have $\operatorname{Im} F_{i} \cap \operatorname{Im} F_{j}=\{0\}$ and $\operatorname{Ker} F_{i_{1}}=\operatorname{Im} F_{i_{2}} \oplus \operatorname{Im} F_{i_{3}}$ where $\left\{i_{1}, i_{2}, i_{3}\right\}=\{1,2,3\}$. Since ${ }^{\circ} A={ }^{\circ} P_{A}$, ${ }^{\circ}(B-A)={ }^{\circ} P_{B-A}$ and $B^{\circ}=P_{B}^{\circ}$, from Theorem 2.4 it follows that $\operatorname{Im} F_{1}=$ $\operatorname{Im} P_{A}=\overline{\operatorname{Im} A}, \operatorname{Im} F_{2}=\operatorname{Im} P_{B-A}=\overline{\operatorname{Im}(B-A)}$ and $\operatorname{Im} F_{3}=\operatorname{Im}\left(I-P_{B}\right)=$ $\operatorname{Ker} P_{B}=\operatorname{Ker} B$. Therefore,

$$
X=\overline{\operatorname{Im} A} \oplus \overline{\operatorname{Im}(B-A)} \oplus \operatorname{Ker} B .
$$

Moreover, since $A^{\circ}=P_{A}^{\circ}$, Theorem 2.4 shows that $\operatorname{Ker} A=\operatorname{Ker} P_{A}=\operatorname{Ker} F_{1}$. But, from $F_{1} F_{2}=0$ and $F_{1} F_{3}=0$ it follows that $\operatorname{Ker} F_{1}=\operatorname{Im} F_{2} \oplus \operatorname{Im} F_{3}$. Thus

$$
\operatorname{Ker} A=\overline{\operatorname{Im}(B-A)} \oplus \operatorname{Ker} B \text {. }
$$

Similarly

$$
\operatorname{Ker}(B-A)=\overline{\operatorname{Im} A} \oplus \operatorname{Ker} B .
$$

We are now in a position to restate Theorem 3.5. The following result is proved in Theorem 1 in [7]. The only difference is that we have $3 \times 3$ matrix representations in contrast to $2 \times 2$ matrix representations obtained in [7]. 
Theorem 3.7. Let $A, B \in I_{X}$. Then the following statements are equivalent:

(1) $A \leq \sharp B$;

(2) $X=\overline{\operatorname{Im} A} \oplus \overline{\operatorname{Im}(B-A)} \oplus \operatorname{Ker} B$ and $A$ and $B$ have the following matrix representations with respect to this decomposition

$$
A=\left[\begin{array}{ccc}
A_{1} & 0 & 0 \\
0 & 0 & 0 \\
0 & 0 & 0
\end{array}\right] \text { and } B=\left[\begin{array}{ccc}
A_{1} & 0 & 0 \\
0 & B_{1} & 0 \\
0 & 0 & 0
\end{array}\right],
$$

where $A_{1}: \overline{\operatorname{Im} A} \rightarrow \overline{\operatorname{Im} A}$ and $B_{1}: \overline{\operatorname{Im}(B-A)} \rightarrow \overline{\operatorname{Im}(B-A)}$ are injective bounded operators with $\overline{\operatorname{Im} A_{1}}=\overline{\operatorname{Im} A}$ and $\overline{\operatorname{Im} B_{1}}=\overline{\operatorname{Im}(B-A)}$.

(3) $A^{2}=A B=B A$.

Theorem 3.8. The relation $\leq \sharp$ is a partial order on $\mathcal{I}_{R}$.

Proof. If $a \in \mathcal{I}_{R}$ then $a=p_{a} a=a p_{a}$, so $\leq \sharp$ is reflexive. Suppose that $a, b \in \mathcal{I}_{R}$ and $a \leq^{\sharp} b, b \leq^{\sharp} a$. We have proved in Lemmas 3.3 and 3.4 that $a \leq^{\sharp} b$ implies $p_{a}=p_{a} p_{b}=p_{b} p_{a}$. In addition we have $p_{b}=p_{a} p_{b}$ so $p_{a}=p_{b}$. Now, $a=p_{a} b=$ $p_{b} b=b$. To show the transitivity suppose that $a, b, c \in \mathcal{I}_{R}$ and $a \leq^{\sharp} b, b \leq^{\sharp} c$. Then $a=p_{a} b=b p_{a}$ and $b=p_{b} c=c p_{b}$. Also, $p_{a} p_{b}=p_{b} p_{a}=p_{a}$. It follows that $a=p_{a} b=p_{a} p_{b} c=p_{a} c$ and $a=b p_{a}=c p_{b} p_{a}=c p_{a}$. By definition, $a \leq^{\sharp} c$.

We now proceed to one-sided sharp orders. See [15] for definitions in complex matrix case.

Definition 3.9. For $a, b \in R$ we write $a \sharp \leq b$ if $a \in \mathcal{I}_{R}$, there exists an idempotent $q$ with $a^{\circ}=q^{\circ}$ and

$$
a=p_{a} b=b q .
$$

Definition 3.10. For $a, b \in R$ we write $a \leq \sharp b$ if $a \in \mathcal{I}_{R}$, there exists an idempotent $q$ with ${ }^{\circ} a={ }^{\circ} q$ and

$$
a=q b=b p_{a} .
$$

The relations $\sharp \leq$ and $\leq \sharp$ are called left-sharp and right-sharp partial order respectively.

Theorem 3.11. Let $a, b \in \mathcal{I}_{R}$. Then the following statements are equivalent:

(1) $a \sharp \leq b$;

$$
a=\left[\begin{array}{ll}
a & 0 \\
0 & 0
\end{array}\right]_{p_{a} \times q} \text { and } b=\left[\begin{array}{cc}
a & 0 \\
0 & b-a
\end{array}\right]_{p_{a} \times q},
$$

where $q$ is some idempotent such that $q^{\circ}=a^{\circ}$.

(3) $a^{2}=a b$ and $a=b q$ for some idempotent $q$ such that $q^{\circ}=a^{\circ}$.

Proof. (1) $\Rightarrow(2)$ : Suppose that $a \sharp \leq b$, that is $a=p_{a} b=b q$ where $q^{\circ}=a^{\circ}$. From $q^{\circ}=a^{\circ}$ we have $a(1-q)=0$ so $a q=a$. Using $a=p_{a} a=a q=p_{a} b=b q$ one can easy check that

$$
a=p_{a} a q, \quad b-a=\left(1-p_{a}\right) b(1-q) .
$$

Since $b=a+(b-a)$, the matrix forms for $a$ and $b$ given in (3.4) follow. 
$(2) \Rightarrow(1)$ : Since $a=p_{a} a=a q$, from (3.4) and

$$
p_{a}=\left[\begin{array}{cc}
p_{a} & 0 \\
0 & 0
\end{array}\right]_{p_{a} \times p_{a}} \text { and } q=\left[\begin{array}{cc}
q & 0 \\
0 & 0
\end{array}\right]_{q \times q},
$$

it follows that $a=p_{a} b=b q$, that is $a \sharp \leq b$.

$(1) \Leftrightarrow(3)$ follows by Lemma 3.3.

In the same manner we can prove the following theorem.

Theorem 3.12. Let $a, b \in \mathcal{I}_{R}$. Then the following statements are equivalent:

(1) $a \leq \sharp b$;

(2)

$$
a=\left[\begin{array}{ll}
a & 0 \\
0 & 0
\end{array}\right]_{q \times p_{a}} \text { and } b=\left[\begin{array}{cc}
a & 0 \\
0 & b-a
\end{array}\right]_{q \times p_{a}},
$$

where $q$ is some idempotent such that ${ }^{\circ} q={ }^{\circ} a$.

(3) $a^{2}=b a$ and $a=q b$ for some idempotent $q$ such that ${ }^{\circ} q={ }^{\circ} a$.

Theorem 3.13. The relations $\sharp \leq$ and $\leq \sharp$ are partial orders on $\mathcal{I}_{R}$.

Proof. We will give the proof only for the left-sharp order because the proof for right-sharp order can be obtained similarly. The reflexivity of $\sharp \leq$ follows by $a=p_{a} a=a p_{a}$. Suppose that $a, b \in \mathcal{I}_{R}$ and $a \sharp \leq b, b \sharp \leq a$. We have $a=p_{a} b=b q$, $b=p_{b} a=a r$ where $q$ and $r$ are idempotents such that $q^{\circ}=a^{\circ}$ and $r^{\circ}=b^{\circ}$. By (3.5) we have

$$
b-a=\left(1-p_{a}\right) b(1-q)=\left(1-p_{a}\right) \operatorname{ar}(1-q)=0 .
$$

Finally, suppose that $a, b \in \mathcal{I}_{R}$ and $a \sharp \leq b, b \sharp \leq c$. By Definition 3.9 we have $a=p_{a} b=b q$ and $b=p_{b} c=c r$ where $q$ and $r$ are idempotents such that $q^{\circ}=a^{\circ}$ and $r^{\circ}=b^{\circ}$. By Lemma 3.3 we have $p_{a} p_{b}=p_{a}$, so

$$
p_{a} c=p_{a} p_{b} c=p_{a} b=a .
$$

Let $q^{\prime}=r q$. Since $r^{\circ}=b^{\circ}$, we have $b(1-r)=0$ and hence $p_{a} b r q=p_{a} b q$. Therefore, $a r q=p_{a} b r q=p_{a} b q=p_{a} a=a$, so $1-r q \in a^{\circ}=q^{\circ}$. Thus, $q(1-r q)=0$ that is $q=q r q$. Consequently, $q^{\prime}$ is an idempotent. From $q^{\prime}=r q$ and $q=q q^{\prime}$ it follows that $q^{\circ}=q^{\circ}=a^{\circ}$. Finally,

$$
c q^{\prime}=c r q=b q=a,
$$

so $a \sharp \leq b$.

\section{Core PARTIAL ORDER IN RINGS}

The star partial order, introduced by Drazin in [6], is defined in a ring $R$ with proper involution $*$ by:

$$
a \leq^{*} b \Longleftrightarrow a a^{*}=b a^{*} \text { and } a^{*} a=a^{*} b .
$$

Recall that involution is proper if for any $a \in R$, $a a^{*}=0$ implies $a=0$. The involution of any Rickart *-ring is proper [3]. In [13], the following definition is given. 
Definition 4.1. Let $R$ be a ring with involution $*$. For $a, b \in R$ we say that $a \leq^{*} b$ if there exist self-adjoint idempotent elements $p, q \in R$ such that

$$
{ }^{\circ} a={ }^{\circ} p, \quad a^{\circ}=q^{\circ}, \quad a=p b=b q .
$$

Since Definition 4.1 considers only those elements $a$ for which there exist selfadjoint idempotents $p$ and $q$ with ${ }^{\circ} a={ }^{\circ} p=R(1-p)$ and $a^{\circ}=p^{\circ}=(1-p) R$, we suppose in this section that $R$ is a Rickart $*$-ring. It is shown in [13] that the star partial order given by Definition 4.1 is equivalent in $R$ with star partial order given by (4.1). For $a \in R$, let us denote by $\operatorname{lp}(a)$ the unique self-adjoint idempotent such that ${ }^{\circ} a={ }^{\circ} \operatorname{lp}(a)$. Similarly, let $\operatorname{rp}(a)$ denote the unique selfadjoint idempotent such that $a^{\circ}=\operatorname{rp}(a)^{\circ}$. It is easy to see that

$$
a=\operatorname{lp}(a) a=\operatorname{arp}(a) .
$$

Beside the star partial order, the left star and right star partial orders on $R$ are defined in [13].

Definition 4.2. For $a, b \in R$ we write $a * \leq b$ if there exists an idempotent $q$ with $a^{\circ}=q^{\circ}$ such that

$$
a=\operatorname{lp}(a) b=b q .
$$

We write $a \leq * b$ if there exists an idempotent $q$ with ${ }^{\circ} a={ }^{\circ} q$ such that

$$
a=q b=\operatorname{brp}(a) \text {. }
$$

The following lemmas enable us to simplify definitions 4.1 and 4.2.

Lemma 4.3. [13] For $a, b \in R$ the following are equivalent:

(1) $a=\operatorname{lp}(a) b$;

(2) $a=p b$, for some self-adjoint idempotent $p$;

(3) $a^{*} a=a^{*} b$.

Lemma 4.4. [13] For $a, b \in R$ the following are equivalent:

(1) $a=\operatorname{brp}(a)$;

(2) $a=b p$, for some self-adjoint idempotent $p$;

(3) $a a^{*}=b a^{*}$.

From lemmas 4.3 and 4.4 it follows that $a \leq^{*} b$ if and only if $a=p b=b q$ for some self-adjoint idempotents $p$ and $q$. Also, $a * \leq b$ if and only if $a=p b=b q$ for some self-adjoint idempotent $p$ and some idempotent $q$ with $a^{\circ}=q^{\circ}$. Likewise, $a \leq * b$ if and only if $a=q b=b p$ for some self-adjoint idempotent $p$ and some idempotent $q$ with ${ }^{\circ} a={ }^{\circ} q$.

Finally, the core matrix partial order, introduced by Baksalary and Trenkler in [1], is defined on the set $I_{1, n}$. One of the equivalent definition is the following (see [11]):

$$
A \leq{ }^{\boxplus} B A^{*} A=A^{*} B \text { and } A A^{\sharp}=B A^{\sharp} .
$$

Thus, the core partial order is "between" star and sharp partial orders. The extension of $\leq \circledast$ from $I_{1, n}$ to $\mathcal{I}_{R}$ is natural.

Definition 4.5. For $a, b \in R$ we write $a \leq \notin b$ if $a \in \mathcal{I}_{R}$ and

$$
a=\operatorname{lp}(a) b=b p_{a} .
$$


Of course, we can also consider the dual core partial order.

Definition 4.6. For $a, b \in R$ we write $a \leq \notin b$ if $a \in \mathcal{I}_{R}$ and

$$
a=p_{a} b=\operatorname{brp}(a) \text {. }
$$

Remark 4.7. In [4] the minus partial order is extended from $\mathcal{B}(H)$, where $H$ is Hilbert space, to an arbitrary Rickart ring:

$a \leq^{-} b \Longleftrightarrow a=p b=b q$, for some idempotents $p$ and $q$ with ${ }^{\circ} a={ }^{\circ} p$ and $a^{\circ}=q^{\circ}$.

We can conclude that if $\leq \in\left\{\leq^{\sharp}, \sharp \leq, \leq \sharp, \leq^{*}, * \leq, \leq *, \leq^{\boxplus}, \leq_{\circledast}\right\}$ then $a \leq b$ implies $a \leq^{-} b$.

Recall the definition of well known partial order on the set of idempotents. If $e$ and $f$ are idempotents than

$$
e \leq f \Longleftrightarrow e=e f=f e .
$$

Suppose that $\preceq \in\left\{\leq^{-}, \leq^{\sharp}, \sharp \leq, \leq \sharp\right\}$ and let $e, f$ be idempotents. Note that $p_{e}=e$. It is not difficult to see that $e \preceq f$ if and only if $e \leq f$. Moreover, if $e$ and $f$ are self-adjoint idempotents then $\operatorname{lp}(e)=\operatorname{rp}(e)=p_{e}=e$. Now one can show that $e \leq f$ if and only if $e \preceq f$ where $\preceq \in\left\{\leq^{*}, * \leq, \leq *, \leq \boxplus, \leq \notin\right\}$. Therefore, all considered orders are extensions of the natural partial order on the set of self-adjoint idempotents.

In what follows we will only consider the core partial order; the properties of dual core partial order can be obtained analogously.

Theorem 4.8. Let $a, b \in R$. Then

$$
a \leq \notin b \Longleftrightarrow a * \leq b \text { and } a \leq \sharp b \text {. }
$$

The relation $\leq \notin$ is a partial order on $\mathcal{I}_{R}$.

Proof. By definitions 3.10, 4.2 and 4.5 it follows that $a \leq \notin b$ if and only if $a * \leq b$ and $a \leq \sharp b$. We have proved in Theorem 3.13 that $\leq \sharp$ is a partial order on $R$. Also, $* \leq$ is a partial order on $R$, see [13]. It follows that $\leq \notin$ is a partial order on $R$.

Theorem 4.9. Let $a, b \in \mathcal{I}_{R}$. Then the following statements are equivalent:

(1) $a \leq \notin b$;

(2) There exists an orthogonal decomposition of the identity of $R$

$$
1=e_{1}+e_{2}+e_{3}
$$

and there exists a decomposition of the identity of $R$

$$
1=f_{1}+f_{2}+f_{3}
$$

such that $e_{1}=\operatorname{lp}(a), e_{2}=\operatorname{lp}(b-a), f_{1}=p_{a}, f_{2}^{\circ}=(b-a)^{\circ}$ and

$$
a=\left[\begin{array}{ccc}
a & 0 & 0 \\
0 & 0 & 0 \\
0 & 0 & 0
\end{array}\right]_{e \times f} \text { and } b=\left[\begin{array}{ccc}
a & 0 & 0 \\
0 & b-a & 0 \\
0 & 0 & 0
\end{array}\right]_{e \times f} \text {. }
$$

(3) $a^{*} a=a^{*} b$ and $a^{2}=b a$. 
Proof. $(1) \Rightarrow(2)$ : Suppose that $a, b \in \mathcal{I}_{R}$ and $a \leq \notin b$. Then $a=\operatorname{lp}(a) b=b p_{a}$. We have $1-\operatorname{lp}(b) \in{ }^{\circ} \operatorname{lp}(b)={ }^{\circ} b$ so

$$
(1-\operatorname{lp}(b)) a=(1-\operatorname{lp}(b)) b p_{a}=0 .
$$

Thus $1-\operatorname{lp}(b) \in{ }^{\circ} a={ }^{\circ} \operatorname{lp}(a)$. We conclude that $\operatorname{lp}(a)=\operatorname{lp}(b) \operatorname{lp}(a)$ and $\operatorname{lp}(a)=$ $\operatorname{lp}(a) \operatorname{lp}(b)$ since $\operatorname{lp}(a)$ and $\operatorname{lp}(b)$ are self-adjoint. Therefore, $\operatorname{lp}(b)-\operatorname{lp}(a)$ is selfadjoint idempotent. To show that

$$
\operatorname{lp}(b-a)=\operatorname{lp}(b)-\operatorname{lp}(a)
$$

it is enough to show that ${ }^{\circ}(b-a)={ }^{\circ}(\operatorname{lp}(b)-\operatorname{lp}(a))$. Using $b=\operatorname{lp}(b) b$ and $a=\operatorname{lp}(a) b$ we obtain

$$
\begin{aligned}
& x(b-a)=0 \Longleftrightarrow x(\operatorname{lp}(b) b-\operatorname{lp}(a) b)=0 \Longleftrightarrow x(\operatorname{lp}(b)-\operatorname{lp}(a)) b=0 \\
& \Longleftrightarrow x(\operatorname{lp}(b)-\operatorname{lp}(a)) \in^{\circ} b={ }^{\circ} \operatorname{lp}(b) \Longleftrightarrow x(\operatorname{lp}(b)-\operatorname{lp}(a)) \operatorname{lp}(b)=0 \\
& \Longleftrightarrow x(\operatorname{lp}(b) \operatorname{lp}(b)-\operatorname{lp}(a) \operatorname{lp}(b))=0 \Longleftrightarrow x(\operatorname{lp}(b)-\operatorname{lp}(a))=0 .
\end{aligned}
$$

It follows that $1=e_{1}+e_{2}+e_{3}$ where $e_{1}=\operatorname{lp}(a), e_{2}=\operatorname{lp}(b-a)=\operatorname{lp}(b)-\operatorname{lp}(a)$, $e_{3}=1-\operatorname{lp}(b)$ is an orthogonal decomposition of the identity of $R$. We have proved in Lemma 3.4 that $a=b p_{a}$ implies $p_{b} p_{a}=p_{a}$. As $1-p_{b} \in p_{b}^{\circ}=b^{\circ}$ we have

$$
a\left(1-p_{b}\right)=\operatorname{lp}(a) b\left(1-p_{b}\right)=0,
$$

so $1-p_{b} \in a^{\circ}=p_{a}^{\circ}$. We conclude that $p_{a}=p_{a} p_{b}$ and thus $p_{b}-p_{a}$ is an idempotent. From $p_{a}=p_{a} p_{b}=p_{b} p_{a}$ we can show that $1=f_{1}+f_{2}+f_{3}$, where $f_{1}=p_{a}, f_{2}=p_{b}-p_{a}, f_{3}=1-p_{b}$, is a decomposition of the identity of $R$. We can show that $(b-a)^{\circ}=\left(p_{b}-p_{a}\right)^{\circ}$ just as in the proof of Theorem 3.5. Let us prove that $a p_{b}=a$. Indeed, from $p_{a}=p_{a} p_{b}$ we obtain $1-p_{b} \in p_{a}^{\circ}=a^{\circ}$ that is $a\left(1-p_{b}\right)=0$. Note that $e_{1} a f_{1}=\operatorname{lp}(a) a p_{a}=a$ and

$$
e_{2} b f_{2}=(\operatorname{lp}(b)-\operatorname{lp}(a)) b\left(p_{b}-p_{a}\right)=(b-a)\left(p_{b}-p_{a}\right)=b\left(p_{b}-p_{a}\right)=b-a .
$$

Thus, $a=e_{1} a f_{1}$ and $e_{2}(b-a) f_{2}=b-a$ so $b=a+(b-a)=e_{1} a f_{1}+e_{2}(b-a) f_{2}$. Since $a$ and $b$ have the unique matrix representations with respect to decompositions of the identity, $1=e_{1}+e_{2}+e_{3}$ and $1=f_{1}+f_{2}+f_{3}$, we conclude that the matrix forms of $a$ and $b$ are as in (4.2).

$(2) \Rightarrow(1)$ : Since $\operatorname{lp}(a)=e_{1} \operatorname{lp}(a) e_{1}$ and $p_{a}=f_{1} p_{a} f_{1}$, from the uniqueness of the representation we obtain

$$
\operatorname{lp}(a)=\left[\begin{array}{ccc}
\operatorname{lp}(a) & 0 & 0 \\
0 & 0 & 0 \\
0 & 0 & 0
\end{array}\right]_{e \times e} \text { and } p_{a}=\left[\begin{array}{ccc}
p_{a} & 0 & 0 \\
0 & 0 & 0 \\
0 & 0 & 0
\end{array}\right]_{f \times f}
$$

Now it is easy to show that $a=\operatorname{lp}(a) b=b p_{a}$ that is $a \leq \notin b$.

$(1) \Leftrightarrow(3)$ follows by lemmas 3.4 and 4.3.

Remark 4.10. We saw in in the proof of Theorem 4.9 that $a \leq \notin b$ implies $\operatorname{lp}(b-$ $a)=\operatorname{lp}(b)-\operatorname{lp}(a)$ and $p_{b-a}^{\circ}=\left(p_{b}-p_{a}\right)^{\circ}$. Let us prove that $a \leq \notin b$ does not imply $p_{b-a}=p_{b}-p_{a}$ in general. Suppose, contrary to our claim, that $a \leq \notin b$ implies $p_{b-a}=p_{b}-p_{a}$. Then by the equivalence of (1) and (2) in Theorem 4.9 we can conclude that $a \leq \circledast b$ if and only if $b-a \leq \circledast b$. But this is not true even in the complex matrix case, see [1]. 
By Theorem 4.9 we can characterize the set of all elements $b$ that are above an element $a \in \mathcal{I}_{R}$ under the core partial order:

$$
a \leq{ }^{\boxplus} b \Longleftrightarrow b=\left[\begin{array}{cc}
a & 0 \\
0 & b_{1}
\end{array}\right]_{\operatorname{lp}(a) \times p_{a}} \Longleftrightarrow b=a+(1-\operatorname{lp}(a)) x\left(1-p_{a}\right),
$$

where $b_{1} \in(1-\operatorname{lp}(a)) R\left(1-p_{a}\right)$ and $x \in R$ are arbitrary.

Remark 4.11. Suppose that $R=\mathcal{B}(H), A, B \in I_{H}$, where $H$ is Hilbert space and let us consider Theorem 4.9. Let $E_{1}=\operatorname{lp}(A), E_{2}=\operatorname{lp}(B-A)=\operatorname{lp}(B)-\operatorname{lp}(A)$, $E_{3}=1-\operatorname{lp}(B)$ and $F_{1}=P_{A}, F_{2}=P_{B}-P_{A}, F_{3}=1-P_{B}$. Since ${ }^{\circ} E_{1}={ }^{\circ} A$, ${ }^{\circ} E_{2}={ }^{\circ}(B-A)$ and ${ }^{\circ} \operatorname{lp}(B)={ }^{\circ} B$, by Theorem 2.4 we obtain $\operatorname{Im} E_{1}=\overline{\operatorname{Im} A}$, $\operatorname{Im} E_{2}=\overline{\operatorname{Im}(B-A)}$ and

$$
\operatorname{Im} E_{3}=\operatorname{Ker} \operatorname{lp}(B)=\operatorname{Ker}(\operatorname{lp}(B))^{*}=(\operatorname{Im} \operatorname{lp}(B))^{\perp}=\overline{\operatorname{Im} B}^{\perp}=\operatorname{Ker} B^{*} .
$$

Since $1=E_{1}+E_{2}+E_{3}$ is orthogonal decomposition of the identity of $\mathcal{B}(H)$ we conclude that $H=\operatorname{Im} E_{1} \oplus^{\perp} \operatorname{Im} E_{2} \oplus^{\perp} \operatorname{Im} E_{3}$ where $\oplus^{\perp}$ denotes orthogonal topological direct sum of subspaces. Therefore,

$$
H=\overline{\operatorname{Im} A} \oplus^{\perp} \overline{\operatorname{Im}(B-A)} \oplus^{\perp} \operatorname{Ker} B^{*} .
$$

Similarly, since ${ }^{\circ} F_{1}={ }^{\circ} A, P_{B}^{\circ}=B^{\circ}$ we obtain $\operatorname{Im} F_{1}=\overline{\operatorname{Im} A}$ and $\operatorname{Im} F_{3}=$ $\operatorname{Ker} P_{B}=\operatorname{Ker} B$. Thus we have

$$
H=\overline{\operatorname{Im} A} \oplus \operatorname{Im}\left(P_{B}-P_{A}\right) \oplus \operatorname{Ker} B .
$$

Furthermore, since $A^{\circ}=F_{1}^{\circ}$ we have

$$
\operatorname{Ker} A=\operatorname{Ker} F_{1}=\operatorname{Im} F_{2} \oplus \operatorname{Im} F_{3}=\operatorname{Im}\left(P_{B}-P_{A}\right) \oplus \operatorname{Ker} B .
$$

Also, from $(B-A)^{\circ}=F_{2}^{\circ}$ we conclude that

$$
\operatorname{Ker}(B-A)=\operatorname{Ker} F_{2}=\operatorname{Im} F_{1} \oplus \operatorname{Im} F_{3}=\overline{\operatorname{Im} A} \oplus \operatorname{Ker} B .
$$

We can now restate Theorem 4.9.

Theorem 4.12. Let $A, B \in I_{H}$, where $H$ is Hilbert space. Then the following statements are equivalent:

(1) $A \leq \boxplus B$;

$$
\begin{aligned}
& H=\overline{\operatorname{Im} A} \oplus \operatorname{Im}\left(P_{B}-P_{A}\right) \oplus \operatorname{Ker} B, \\
& H=\overline{\operatorname{Im} A} \oplus^{\perp} \overline{\operatorname{Im}(B-A)} \oplus^{\perp} \operatorname{Ker} B^{*}
\end{aligned}
$$

and

$A, B: \overline{\operatorname{Im} A} \oplus \operatorname{Im}\left(P_{B}-P_{A}\right) \oplus \operatorname{Ker} B \rightarrow \overline{\operatorname{Im} A} \oplus^{\perp} \overline{\operatorname{Im}(B-A)} \oplus^{\perp} \operatorname{Ker} B^{*}$ have the following matrix representations

$$
A=\left[\begin{array}{ccc}
A_{1} & 0 & 0 \\
0 & 0 & 0 \\
0 & 0 & 0
\end{array}\right] \text { and } B=\left[\begin{array}{ccc}
A_{1} & 0 & 0 \\
0 & B_{1} & 0 \\
0 & 0 & 0
\end{array}\right]
$$

where $A_{1}: \overline{\operatorname{Im} A} \rightarrow \overline{\operatorname{Im} A}$ and $B_{1}: \operatorname{Im}\left(P_{B}-P_{A}\right) \rightarrow \overline{\operatorname{Im}(B-A)}$ are injective bounded operators with $\overline{\operatorname{Im} A_{1}}=\overline{\operatorname{Im} A}$ and $\overline{\operatorname{Im} B_{1}}=\overline{\operatorname{Im}(B-A)}$. 
(3) $A^{*} A=A^{*} B$ and $A^{2}=B A$.

Acknowledgement. The author would like to thank the anonymous reviewer for her/his constructive comments that improved the presentation of this paper. The author is supported by the Ministry of Science, Republic of Serbia, grant no. 174007.

\section{REFERENCES}

1. O.M. Baksalary and G. Trenkler, Core inverse of matrices, Linear Multilinear Algebra 58 (2010), no. 6, 681-697.

2. A. Ben-Israel and T.N.E. Greville, Generalized Inverses: Theory and Applications, 2nd Edition, Springer, New York, 2003.

3. S.K. Berberian, Baer * ${ }^{*}$-rings, Springer-Verlag, New York, 1972.

4. D.S. Djordjević, D.S. Rakić and J. Marovt, Minus partial order in Rickart rings, preprint (available at http://www.imfm.si/preprinti/index.php?langID=1).

5. D.S. Djordjević and V. Rakočević, Lectures on generalized inverses, Faculty of Scince and Mathematics, University of Niš, 2008

6. M.P. Drazin, Natural structures on semigroups with involution, Bull. Amer. Math. Soc. 84 (1978), 139-141.

7. M.A. Efimov, On the $\stackrel{\#}{\leq}$-order on the set of linear bounded operators in Banach Space, Math. Notes 93 (2013), no. 5, 784-788.

8. R.E. Hartwig, How to partialy order regular elements, Math. Japon. 25 (1980), 1-13.

9. N. Jacobson, Structure of rings, Amer. Math. Soc., 1968.

10. I. Kaplansky, Rings of operators, W. A. Benjamin, 1968.

11. S.B. Malik, L. Reuda and N. Thome, Further properties on the core partial order and other matrix partial orders, Linear Multilinear Algebra 62 (2014), no. 12, 1629-1648.

12. J. Marovt, On partial orders in Rickart rings, Linear Multilinear Algebra, DOI: 10.1080/03081087.2014.972314

13. J. Marovt, D.S. Rakić and D.S. Djordjević, Star, left-star, and right-star partial orders in Rickart*-rings, Linear Multilinear Algebra 63 (2015), no. 2, 343-365.

14. S.K. Mitra, On Group Inverses and the Sharp Order, Linear Algebra Appl. 92 (1987), 17-37.

15. S.K. Mitra, P. Bhimasankaram and S.B. Malik, Matrix partial orders, shorted operators and applications, World Scientific, 2010.

16. P. Šemrl, Automorphisms of $B(H)$ with respect to minus partial order, J. Math. Anal. Appl. 369 (2010), 205-213.

Faculty of Mechanical Engineering, University of Niš, Aleksandra Medvedeva 14, 18000 Niš, SERBIA.

E-mail address: rakic.dragan@gmail.com 